\title{
Correlation between N/O nebular ratio and central star mass: distances to galactic PN
}

\section{J. O. Cazetta and W. J. Maciel}

Instituto Astronômico e Geofísico da USP, São Paulo, Brazil

In the planetary nebulae (PNe) studies, their distance determination remains a confusing issue. The statistical distance scales cannot be regarded as reliable, and there are few PNe with individual distance determinations. In this work, we present a distance scale based on the method of individual distance determination by Méndez et al. (1988, A\&A $190,113)$.

The distance can be written as a function of the central star parameters: $F(\lambda 5480), g$, $\mathbf{M}_{v}$ and $\mathbf{M c}$. There is a large uncertainty in the prediction of the core mass Mc. We propose a relationship between $\mathrm{Mc}$ and N/O nebular ratio which allows us to obtain a new distance scale for PNe. (FAPESP, CNPq) 